\title{
Performance Assessment of Swarm Robots
}

\author{
E. Oztemel ${ }^{1}$, C. Kubat ${ }^{2}$, O. Uygun ${ }^{2}$, T. Canvar ${ }^{2}$, T. Korkusuz ${ }^{2}$, V. Raja ${ }^{3}$, \\ and A. Soroka ${ }^{4}$ \\ ${ }^{1}$ Marmara University, Eng. Fac., Dept. Of Ind. Eng., Göztepe Campus, Istanbul \\ ${ }^{2}$ Sakarya University, Eng. Fac., Dept. Of Ind. Eng., Esentepe Campus, Sakarya \\ ${ }^{3}$ University of Warwick, Warwick Manufacturing Group, Warwick, UK \\ ${ }^{4}$ Cardiff University, Manufacturing Engineering Center, Cardiff, UK
}

\begin{abstract}
Swarm intelligence is the emergent collective intelligence of groups of simple autonomous agents which are autonomous subsystems that interact with their environment. This paper presents a performance evaluation system for swarm robots. The model proposed includes a set of performance assessment criteria and performance assessment and monitoring system. The proposed approach is developed for swarm robots developed for health system responsible for delivery, guidance, monitoring, recognition, and delivery which a project in European $6^{\text {th }}$ Framework Research Program and carried out by several European nations.
\end{abstract}

Keywords: swarm robots, performance assessment.

\section{Introduction}

Swarm intelligence is the emergent collective intelligence of groups of simple autonomous agents which are autonomous subsystems that interact with their environment, which probably consists of other agents, but act relatively independently from all other agents [8]. IT is important to point out the concept of swarm here as it provides several advantageous over being a single agent performing certain functions. By creating swarm of robots for example, it may be possible to assign a very complex set of tasks to a set of robots capable of performing collective and cooperative actions. As clearly pointed out by Kassabalidis, et al. [7], swarm intelligence, as demonstrated by natural biological swarms, has numerous powerful properties desirable in many engineering systems. In addition, new paradigms for designing autonomous and scalable systems may result from analytically understanding and extending the design principles and operations exhibited by intelligent biological swarms. A key element of future design paradigms will be emergent intelligence - simple local interactions of autonomous swarm members, with simple primitives, giving rise to complex and intelligent global behaviour.

Swarm intelligence boasts a number of advantages due to the use of mobile agents and stigmergy [7]. These are;

- Scalability: Population of the agents can be adapted according to the network size. Scalability is also promoted by local and distributed agent interactions.

- Fault tolerance: Swarm intelligent processes do not rely on a centralized control mechanism. Therefore the loss of a few nodes or links does not result in catastrophic failure, but rather leads to graceful, scalable degradation. 
- Adaptation: Agents can change, die or reproduce, according to network changes.

- Speed: Changes in the network can be propagated very fast, in contrast with the Bellman-Ford algorithm.

- Modularity: Agents act independently of other network layers.

- Autonomy: Little or no human supervision is required.

- Parallelism: Agent's operations are inherently parallel.

Detailed information about swarm intelligence and their possible characteristics can be found in $[1,3,4,10]$ However, designing a self-organizing swarm robot is not a trivial task. From an engineering perspective, the design problem is generally decomposed into two different phases: (i) the behaviour of the system should be described as the result of interactions among individual behaviours, and (ii) the individual behaviours must be encoded into controllers [5]. Robots are designed to perform specific behaviour. Performance of the robot is assessed in accordance with the capability of performing intended function as effectively as expected. The scope of this Paper is to provide a general framework to indicate basic issues regarding performance assessment and possible implications.

Although there have been many studies on swarm intelligence, there is little information in the literature on a set of criteria to evaluate a swarm intelligence. Rybski et al. [9] showed how the performance is affected by the number of robots working in the same area, the distribution of the targets the robots are searching for, and the complexity of the environment as the environmental factors. Liu and Passino [8] mention to make an evaluation based on the flexibility, which is essentially a robustness property. They proposed measures of fault tolerance and local superiority as indices. They compared two swarm intelligent systems via simulation with respect to these two indices. Similarly, Banks et.al. [3] identified five basic principles that could be used to identify swarm intelligence especially for Unmanned Air Vehicles (UAVs) as follows;

- Proximity. All swarm members should be able to perform elementary space and time computations. That is an attempt to compute an immediate course of action that will have the highest likelihood of achieving the group goal.

- Quality. The swarm members should be able to respond to quality measures with respect to the attainment of their individual goals.

- Diversity of Response. The swarm should not excessively commit its resources in a manner that would leave it vulnerable.

- Stability. This principle is important, because an unstable swarm, that is one that changes its behaviour too often, is inefficient.

- Adaptability. All the swarm members should be able to switch to a different mode of operation, but only when there is a sufficient return on investment.

Xie et al. [11] studied optimizing semiconductor devices by self-organizing particle swarm in their studies. The testing on benchmark functions and an application example for device optimization with designed fitness function is used to assess the performance of the swarm behaviour. Baldassarre et.al. [2] presented a robust solution to a difficult coordination problem that might also be encountered by some organisms, caused by the fact that the robots have to be capable of moving in any direction while being physically connected. And they assess performance of moving capability of moving as quickly as possible and as fast straight as possible, avoiding obstacles, and approaching a light target. Fukuda et al. [6] try to make an evaluation of swarm 
intelligent system based on the flexibility, which is essential, a robustness property. They proposed measures of fault tolerance and local superiority as indices. The approaches implemented in these studies are rather application depended. There is a need for a general framework for creating a swarm robot assessment system. This paper presents such a framework which will be implemented in assessing the performance of swarm robots responsible for cleaning, surveillance, monitoring, delivery and guidance in a hospital.

\section{Proposed Performance Evaluation Model}

Figure 1 indicates the architecture and components of proposed evaluation system. This system is mainly developed for IWARD and European 6th Framework Research Program developed for Intelligent Robot Swarm for Attendance, Recognition, Cleaning and Delivery.

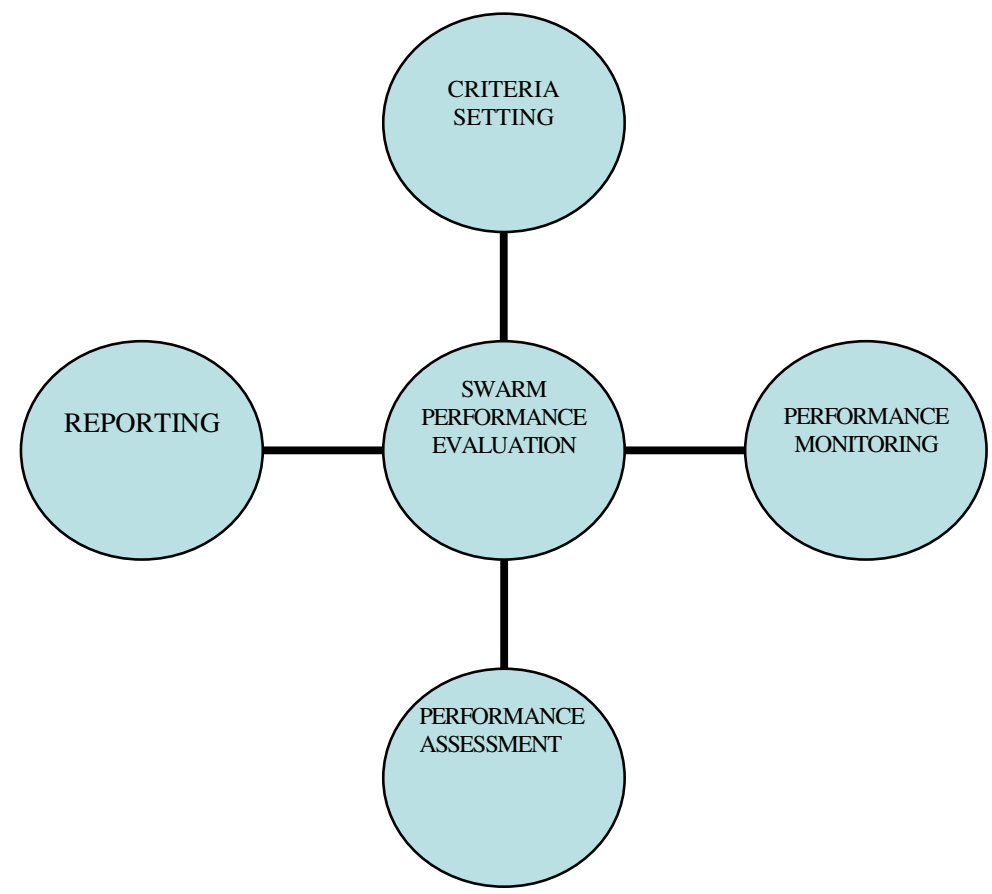

Fig. 1. Elements of performance evaluation system of swarm robots

\section{Criteria Setting}

In order to be able to measure and quantify the performance of swarm robots, a set of criteria needs to be set up. There could be two types of criteria mainly;

- General criteria

- Domain specific criteria 
A set of performance criteria so called indicators are defined taking the following aspect into account.

- Feasibility: Performance indicators should be feasible in terms of cost, risk, technology and social possibilities.

- Manageability: Performance indicator should be targeted at specific functions which can be easily carried out without violating operational rules.

- Usefulness: Performance indicators should be targeted on the functions that provide certain benefit(s) to the user

- Acceptability: Performance indicators should not create any objection by the users.

- Necessity: Performance indicator may be targeted on the desired function which may not also directly related to end user but is needed to perform user related activities

Based on these aspects, a set of general criteria are defined as the following.

- Criterion 1: Functionality of individual robots to make sure that each robot is able to perform operations as expected. $15 \mathrm{sub}$ criteria are defined to measure the functionality of a set of health robots so called IWARD robots, a European $6^{\text {th }}$ framework research project. An example of such a criterion for a robot responsible to deliver an object could be

- Criteria 1a- To deliver the objects to the target destination as much correctly as possible (performance indicator: number of delivery to the wrong destinations, Target: 0 )

- Criterion 2: Robustness (robust behaviour): This criteria is defined to test the ability of the robots to perform allocated tasks as much efficiently, effectively and robust as possible. 16 sub criteria to assess the robustness of IWARD robots are defined, an example of which is

- Criteria 2f- Detection of intervention within the boundaries of sensors (lifting / tipping / tampering) (performance indicator: number of cases unrecognised, target: 0 )

- Criterion 3- Communication skill and information exchange: This criterion is defined to test the ability to transmit and share correct information required by the staff as well as other robots at the correct times. 12 sub criteria to assess the communication performance of IWARD robots are defined, an example of which is;

- Criteria 3d- To transmit information in a secure way (performance indicator: number of unauthorized accesses, Target: 0 )

- Criterion 4- Timeliness and responsiveness: This criterion is defined to test the ability to perform the tasks on time. The following example can be a good indicator for robot performance. 10 sub criteria is designed for IWARD robots for this criterion. An example of a sub criteria is;

- Criteria 4b- Preventing time delays in robots' schedules assigned to the robot (performance indicator: average amount of time delays; Target: 0 $\min )$ 
- Criterion 5- Synchronization: This criterion is defined to test the ability to perform joint actions on time between the robot and other robots, staff and patients. 6 criteria is defined for IWARD robot synchronisation.

- Criterion 5g- Ability to work together with other robots (Performance indicator: tester's assessment, Target: Tester's assessment)

- Criteria 6-Swarm intelligence: This criterion is defined to test the ability to perform intelligent (swarm) behaviour of the robot. IWARD robots are assessed with 14 sub criteria for this.

- Criterion 6h- Capability of mutual decision making (performance indicator: satisfying pass criteria, Target: to be defined during design)

Similar examples can be derived for the following criteria which were identified for swarm robots to assure their performance for their intended functions.

- Criteria 7- Reliability: This criterion is defined to test the ability to perform reliable actions. IWARD robots are assessed with 6 sub criteria for reliability.

- Criteria 8-Usability: This criterion is defined to test the extent to which a product can be used by specified users to achieve specified goals with effectiveness, efficiency and satisfaction in a specified context of use. 10 criteria is defined for IWARD robots for assessing usability.

- Criteria 9-Accessibility: This criterion is defined to test the ability to access that the information is available to the widest possible users.

There could be some domain specific performance criteria related to the activities and users of the swarm robots. For IWARD robots especially end-user comfort and psychological effects on the patients and hospital staff are considered along this line.

\section{Performance Monitoring and Assessment}

Once the set of criteria is defined, they are monitored during the operations of robots. A coloureds scheme can be implemented on a grid indicating performance gaps. There could be a coloured scheme indicating the level of compliance as given below.

- Green colour- respective criteria are fully satisfied

- Blue colour- respective criteria are partially satisfied

- Red colour - respective criteria are not implemented

Figure 2 indicates a performance grid which is designed to monitor the performance of swarm robots. A fully green performance grid indicates an ideal well performing set of robots. Note that Figure 2 only shows criteria listed above. The actual grid will have all criteria with respect to all robots.

Figure 2 indicates that robot 1 has some problems in transferring the information in a secure way. Similarly, Robot 2 has problems with corporate decision making.

To be able to define the colour of the grid for a specific robot for specific criteria an assessment form is designed. It includes a set of facts and questions to be filled out by the assessor which will yield to the colour of the grid. Each assessment sheet is designed based on a scenario defined by the development team to indicate all implications of the domain with respect to delivery, recognition, guidance, cleaning etc. 


\begin{tabular}{|l|l|l|l|}
\hline & \multicolumn{3}{|c|}{ Robots } \\
\hline Performance indicators & R1 & R2 & R3 \\
\hline Criterion 1a & & & \\
\hline Criterion 2f & & & \\
\hline Criterion 3d & & & \\
\hline Criterion 4b & & & \\
\hline Criterion 5g & & & \\
\hline Criterion 6h & & & \\
\hline Criterion .. & & & \\
\hline Criterion .. & & & \\
\hline Criterion .. & & & \\
\hline$\ldots .$. & & & \\
\hline
\end{tabular}

Fig. 2. Performance grid

\section{Conclusion}

This paper presented a performance evaluation system which is developed to assess the performance of swarm robots. A set of criteria is defined based on through an extensive domain analysis and a well defined procedure to make sure that each criteria is acceptable to the users and measurable as well as assuring benefits of the swarm system.

The system is developed for IWARD (Intelligent Robot Swarm for Attendance, Recognition, Cleaning and Delivery) project. The IWARD robots are developed and in the stage of integration. Once the system is integrated and ready to use, then it will be tested and performance of the robots will be identified through implementing the criteria recommended.

There have been several scenarios defined to test the functionality of the robot. An assessment sheet is developed for each scenario. The assessment sheet includes a set of questions and observations to be filled out. These are defined using the set of criteria explained above. Each criteria is detailed down to some measurable and observable statements and facts. Once the robots are assessed based on these facts the colour of respective criteria for each robot will be defined and performance grid will be filled out. This will clearly indicate performance gaps which could then be fixed before hacving the robots in operation.

\section{References}

1. Baldassarre, G., Domenico, P., Nolfi, S.: Coordination and Behaviour Integration in Cooperating Simulated Robots (2004),

http://laral.istc.cnr.it/baldassarre/publications/2004BaldPa riNolf-InteConfSimuAdapBeha-CoorBehaInteInSimuRobo.pdf 
2. Baldassarre, G., Parisi, D., Nolfi, S.: Distributed Coordination of Simulated Robots Based on Self-Organisation, Institute of Cognitive Sciences and Technologies, National Research Council (ISTC-CNR) (2005),

http://laral.istc.cnr.it/baldassarre/publications/2005BaldPa riNolf-ArtiLife-DistCoorInSimuRoboBaseonSelforga.pdf

3. Banks, A., Vincent, J., Phalp, K.: Particle swarm guidance system for autonomus unmanned aerial vehicles in an air defence role. The journal of Navigation 61, 9-29 (2008)

4. Bayındır, L., Şahin, E.: A review of studies in Swarm Robotics. Turk. J. Elec. Engin. 15(2) (2007)

5. Dorigo, M., Trianni, V., Şahin, E., Grob, R., Labella, T.H., Baldassarre, G., Nolfi, S., Deneubourg, J.-L., Mondada, F., Florena, D., Gambardella, L.M.: Evolving SelfOrganizing Behaviors for a Swarm-bot (2004), http: / /www.idsia.ch/ luca/swarmbot-control.pdf

6. Fukuda, T., Funato, D., Sekiyam, K., Arai, F.: Evaluation on flexibility of swarm inelligent system. In: Proceedings of the 1998 IEEE International Conference on Robotics and Automation, May 16-20, vol. 4, pp. 3210-3215 (1998)

7. Kassabalidis, I., El-Sharkawi, M.A., Marks, R.J., Arabshahi, P., Gray, A.A.: Swarm intelligence for routing in communication networks (2001), http: / / ieeexplore. ieee. org/stamp/stamp.j.sp? arnumber $=00966355$

8. Liu, Y., Passino, K.M.: Swarm Intelligence: Literature Overview (2000), http: / /www. ece.osu.edu/ passino/swarms.pdf

9. Rybski, P., Larson, A., Lindahl, M., Gini, M.: Performance Evaluation of Multiple Robots in a Search and Retrieval Task. In: Workshop on Artificial Intelligence and Manufacturing (1998)

10. Takemura, Y., Sato, M., Ishii, K.: Toward realization of swarm intelligence mobile robots. International Congress Series, vol. 1291, pp. 273-276 (2006)

11. Xie, X.-F., Zhang, W.-H., Bi, D.-C.: Optimizing Seöiconductor Devices by Selforganizing Particle Swarm. In: Congres on Evolutionary Computation-CEC, Oregon, USA (2004) 